\title{
Stenosis in laparoscopic gastric bypass: management by endoscopic dilation without fluoroscopic guidance
}

\author{
Jesús Espinel ${ }^{1}$, José Luis de-la-Cruz², Eugenia Pinedo ${ }^{3}$, José Canga² and Felipe de-la-Cruz ${ }^{4}$ \\ ${ }^{1}$ Unit of Endoscopy. Department of Gastroenterology. Hospital de León. León, Spain. Endoscopy Unit. Clínica San \\ Francisco. León, Spain. ${ }^{2}$ Unit of Laparoscopic Surgery. Clínica San Francisco. León, Spain. ${ }^{3}$ Department of Radiology. \\ Hospital de León. León, Spain. ${ }^{4}$ Service of General and Digestive Surgery. Hospital 12 de Octubre. Madrid, Spain
}

\begin{abstract}
Objectives: gastric bypass is the surgical procedure that is carried out most frequently in the treatment of morbid obesity. Stenosis of the gastro-jejunal anastomosis is a relatively frequent complication that requires endoscopic management. However, the optimal dilation technique is yet to be determined. The purpose of this study was to evaluate the safety and efficacy of dilation with a hydrostatic balloon (CRE) without radioscopic guidance in morbidly obese patients treated by laparoscopic bypass.

Material and methods: retrospective review of the data elicited from 525 patients treated against morbid obesity with laparoscopic gastric bypass from January, 2006 to November, 2010.

Results: a total of $22 / 525$ patients $(4.1 \%)$ developed stenosis of the anastomosis [20 women (91\%), 2 men (9\%)]. In four patients $(18.2 \%)$, there was an associated anastomotic ulcer, and in one case, there was a history of bleeding of an ulcer treated with sclerosis one month earlier. The diagnosis of stenosis was done in most patients during the first 90 days after the bypass. All cases were resolved by means of endoscopic dilation without radioscopic guidance, 15 cases $(68.1 \%)$ required a single session, 6 cases $(27.2 \%)$ two sessions, and 1 case $(4.5 \%)$ required four sessions. This last case had an associated anastomotic ulcer. The diameter of the balloons ranged from 12 to $20 \mathrm{~mm}$, generally using diameters of $12-15 \mathrm{~mm}$ in the first session, and increasing them in the following sessions according to the previous result. One patient treated with a $20 \mathrm{~mm}$ balloon presented with a small tear, without showing any evidence of leak of contrast medium in the radioscopic guidance, and was thus managed conservatively. In the follow-up, no re-stenoses were detected.

Conclusions: in our experience, stenosis of the anastomosis in the laparoscopic gastric bypass is an infrequent complication. When it happens, dilation with a hydrostatic balloon is an effective and safe treatment. Radioscopic guidance during dilation is not strictly necessary if norms of progressive dilation are followed.
\end{abstract}

Received: 21-03-11.

Accepted: 29-04-11.

Correspondence: Jesús Espinel Díez. Unit of Endoscopy. Department of Gastroenterology. Hospital de León. C/ Altos de Nava, s/n. 24071 León, Spain. e-mail: espinel.jesus@gmail.com
Key words: Gastric bypass. Gastro-jejunal anastomosis. Hydrostatic balloon dilation. Stricture. Endoscopic dilation. Fluoroscopic guidance. Stenosis of the anastomosis. Obesity.

Espinel J, De-la-Cruz JL, Pinedo E, Canga J, De-la-Cruz F. Stenosis in laparoscopic gastric bypass: management by endoscopic dilation without fluoroscopic guidance. Rev Esp Enferm Dig 2011; 103: 508-510.

\section{INTRODUCTION}

Obesity has become one of the main health problems in industrialized countries. In Spain, half of the adults suffer from overweight and around $20 \%$ are obese (1). This XXI century pandemic has important consequences, such as the increased morbidity and mortality in these patients. Bariatric surgery represents an effective therapeutic option in patients with morbid obesity (a body mass index, BMI $\geq 40$ ), and among these options, gastric bypass is the bariatric surgery that is carried out most frequently. Over the last years, laparoscopic bypass has undergone great development. This route offers clear advantages compared to open surgery, such as: less blood loss during surgery, less postoperative pain, a lower incidence of wound infections, a shorter hospital stay, and a shorter period of recovery (2). The stenosis of the stoma (stenosis of the gastro-jejunal anastomosis) occurs in approximately $3-12 \%$ after gastric bypass, and must be suspected when the patient experiences dysphagia, nausea and vomiting (2-9). Endoscopic dilation of the stenosis by means of a balloon or bougie is considered the treatment of choice, even if in most studies it is not quite clear whether it should be done with or without a radioscopic guidance (4-6,9-18). 


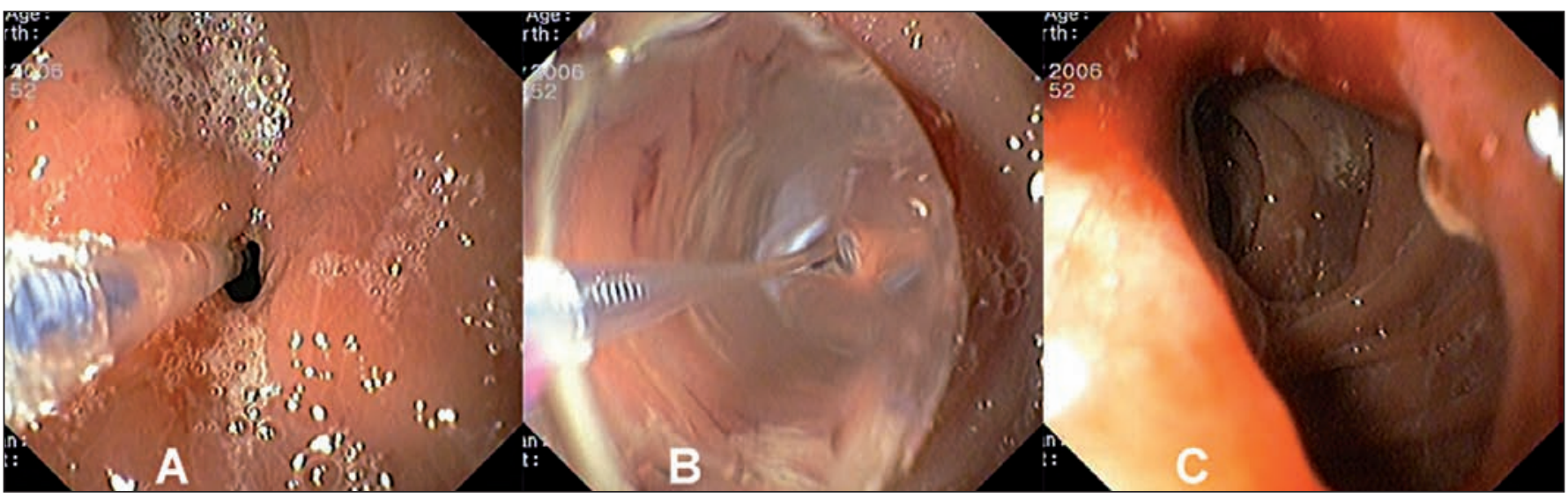

Fig. 1. A. Stenosis of the gastro-jejunal anastomosis. B. Dilation of the stenosis with a $15 \mathrm{~mm}$ TTS (through-the-scope) balloon. C. Gastro-jejunal anastomosis after the dilation.

In this study, we informed of our experience in the management of the stenosis of the stoma in patients with laparoscopic gastric bypass by means of endoscopic dilation with a hydrostatic balloon, without fluoroscopic guidance.

\section{PATIENTS AND METHODS}

Retrospective review of the data elicited prospectively from all the laparoscopic gastric bypass procedures carried out by a same surgeon (J.L.C.V.) from January, 2006 to November, 2010. The surgical technique was the following: five trocars $(10-12 \mathrm{~mm})$ were generally used. The jejunum was sectioned at $40 \mathrm{~cm}$ from the Treitz angle. The feeding loop was measured to be between 100 and $150 \mathrm{~cm}$, according to the body mass index. The Y-en-Roux jejunojejunal anastomosis was carried out with a 45/2.5 stapler, and was finished with a manual, continuous, reabsorbable suture. The gastro-jejunal anastomosis was carried out, with a 30/3.5 stapler, transversely and on the posterior side of the gastric bag's distal end, finishing it with a continuous reabsorbable suture and calibrating it with the $34 \mathrm{~F}$ probe. Finally, water tightness was verified with methylene blue, an aspiration drain was placed between the bag and the gastric remnant, withdrawing the trocars and verifying hemostasis.

Patients who presented with symptoms of dysphagia, nausea or vomiting after the bypass underwent an endoscopy with sedation (midazolam and petidine or propofol). Stenosis of the stoma was considered if passage of the standard endoscope $(9.8 \mathrm{~mm})$ (videoendoscope Olympus) was hindered. The size of the stenosis was estimated by the endoscopist (J.E.), and dilation was carried out with a diameter that was deemed safe. All stenoses were dilated with a dilating TTS balloon (through-the-Scope) (CRE Wire guided Balloon Dilator, Boston S.C.) (Fig. 1). No fluoroscopic guidance was used in any patient. After dilation, patients were advised to call or schedule an appointment if their symptoms persisted.

\section{RESULTS}

Five hundred twenty five (525) patients underwent a laparoscopic gastric bypass. None needed reconversion to open laparotomy. The mean preoperative body mass index was $45.7 \mathrm{~kg} / \mathrm{m}^{2}$. Twenty seven percent $(27 \%)$ of the patients had a BMI > 50 (super obese). The mean age of the patients was of 37.5 years (range: $21-52$ ). A total of 22/525 patients (4.1\%) developed stenosis of the anastomosis [20 women (91\%), 2 men (9\%)]. In four patients (18.2\%), there was an associated anastomotic ulcer, and in one case, there was a history of bleeding ulcer treated with sclerosis one month earlier. The time between the moment the bypass was carried out and the diagnosis ranged between 26 and 768 days (mean: 126 days, median: 60 days), the majority being diagnosed within the first 90 days. All cases were resolved by means of endoscopic dilation without radioscopic guidance, 15 cases $(68.1 \%)$ required a single session, 6 cases $(27.2 \%)$ two sessions, and 1 case $(4.5 \%)$ required four sessions (Table I). This last case had an associated anastomotic ulcer. The diameter of the balloons ranged from 12 to $20 \mathrm{~mm}$, generally using diameters of $12-15 \mathrm{~mm}$ in the first session,

\section{Table I}

\begin{tabular}{ll}
\hline Characteristics & Value \\
\hline $\mathrm{n}$ & $22 / 525(4.1 \%)$ \\
Gender (male/female) & $2 / 20$ \\
Anastomotic ulcer & $4(18.2 \%)$ \\
Endoscopic resolution & $22(100 \%)$ \\
- 1 session & $15(68.1 \%)$ \\
- 2 sessions & $6(27.2 \%)$ \\
- 4 sessions & $1 *(4.5 \%)$ \\
Complications & $1(4.5 \%)$ \\
Re-stenosis during follow-up & $0(0 \%)$ \\
\end{tabular}

*Associated anastomotic ulcer 
and increasing them in the following sessions according to the previous result. One patient treated with a $20 \mathrm{~mm}$ balloon presented with a small tear, without showing any evidence of leak of contrast medium in the radioscopic guidance, and was thus managed conservatively. In the follow-up (mean: 27 months), no re-stenoses were detected.

\section{DISCUSSION}

In spite of the warnings about the growing incidence of obesity, it continues to rise in industrialized countries. One of the main therapies of morbid obesity is bariatric surgery, which has shown good long-term results. Laparoscopic bypass has clear advantages over open surgery, however, the possibility remains, in a variable percentage, of the appearance of stenosis of the gastro-jejunal anastomosis. The pathophysiological mechanisms involved in the formation of stenosis are not well known, although situations such as stoma ulcer, reflux, ischemia of the suture, retraction of the scar, or an inadequate technique, may contribute to its appearance $(2,5)$. Considering the uncertainty of the pathophysiology of stenosis, as a lesser evil is to know that it is an infrequent complication and that the endoscopic treatment achieves a correct and quick resolution of the problem, in most cases, without the need for another surgical intervention.

The optimal method for endoscopic dilation of stomal strictures remains to be determined. Some authors have obtained good results by Savary-Gilliard bougies (11). Although most prefer the use of through-the-scope (TTS) $(4,5,8,10,12,14-18)$. Our experience shows that the dilation of the stenosis of the stoma using TTS balloon is safe and effective. In our study, $68.1 \%$ of the patients were treated successfully with a simple dilation, and $95.3 \%$ with 1 or 3 sessions. Only 1 patient, who had an associated anastomotic ulcer, required 4 sessions. The rational use of dilators, according to the characteristics of the stenosis, allowed these good results. No significant complications were seen during the procedure. The majority of the initial dilations, in spite of being millimetric stenoses, were carried out with balloons of 12-15 $\mathrm{mm}$. This demonstrates the safety of the dilator treatment, in spite of the dilation initiating at high gauges, and the relative low resistance of the fibrosis that conditions the stoma's stenosis. An aspect that we want to highlight in our study is that all the endoscopic dilations were carried out without fluoroscopic guidance. Published studies are not clear when it comes to describing this aspect. The minority of them clearly manifest not using fluoroscopy during dilation $(4,18)$, others claim to have used it in all or in one of their patients $(5,6,8,11,16)$, and finally, others do not make any sort of comment in this regard in their publications $(9,10,14,15,17)$. Our experience demonstrates that carrying out dilations in patients with stenosis of the anastomosis in the gastric bypass is possible without a fluoroscopic guidance, allowing carrying out the technique in the simplest manner, in the same endoscopy room, without radiation for the patient or for the medical staff, and probably for a shorter period of time.

In conclusion, stenosis of the gastro-jejunal anastomosis in laparoscopic gastric bypass is an infrequent complication. Endoscopy allows for its diagnosis and treatment, surpassing carrying out reinterventions. Carrying out a rational dilation allows, in most cases, for the initial management of stenosis with dilators around $15 \mathrm{~mm}$, without the need for radioscopic guidance, and without significant complications.

\section{REFERENCES}

1. OECD Health data 2010.

2. Cruz Vigo F, Cruz Vigo JL. Stenosis in gastric bypass for morbid obesity. Rev Esp Enferm Dig 2010;102:151-8.

3. Sanyal AJ, Sugerman HJ, Kellum JM, Engle KM, Wolfe L. Stomal complications of gastric bypass: incidence and outcome of therapy. Am J Gastroenterol 1992;87:1165-9.

4. Ahmad J, Martin J, Ikramuddin S, Schauer P, Slivka A. Endoscopic balloon dilation of gastroenteric anastomotic stricture after laparoscopic gastric bypass. Endoscopy 2003;35:725-8.

5. Peifer KJ, Shiels AJ, Azar R, Rivera RE, Eagon JC, Jonnalagadda S. Successful endoscopic management of gastrojejunal anastomotic strictures after Roux-en-Y gastric bypass. Gastrointest Endosc 2007;66:24852 .

6. Nguyen NT, Stevens CM, Wolfe BM. Incidence and outcome of anastomotic stricture after laparoscopic gastric bypass. J Gastrointest Surg 2003;7:997-1003.

7. Huang CS. The role of the endoscopist in a multidisciplinary obesity center. Gastrointest Endosc 2009;70:763-7.

8. Barba CA, Butensky MS, Lorenzo M, Newman R. Endoscopic dilation of gastroesophageal anastomosis stricture after gastric bypass. Surg Endosc 2003;17:416-20

9. Podnos YD, Jimenez JC, Wilson SE, Stevens CM, Nguyen NT. Complications after laparoscopic gastric bypass: a review of 3464 cases. Arch Surg 2003;138:957-61.

10. Campillo-Soto A, Torralba-Martínez JA, Martín-Lorenzo JG, LirónRuiz R, Bento-Gerard M, Pérez-Cuadrado E, et al. Gastrojejunal anastomosis stricture after laparoscopic gastric bypass. Our experience with 62 patients. Rev Esp Enferm Dig 2010;102:187-92.

11. Fernández-Esparrach G, Bordas JM, Llach J, Lacy A, Delgado S, Vidal $\mathrm{J}$, et al. Endoscopic dilation with Savary-Gilliard bougies of stomal strictures after laparoscopic gastric bypass in morbidly obese patients. Obes Surg 2008:18:155-61.

12. Barba CA, Butensky MS, Lorenzo M, Newman R. Endoscopic dilation of gastroesophageal anastomosis stricture after gastric bypass. Surg Endosc 2003;17:416-20.

13. Caro L, Sánchez C, Rodríguez P, Bosch J. Endoscopic balloon dilation of anastomotic strictures occurring after laparoscopic gastric bypass for morbid obesity. Dig Dis 2008;26:314-7.

14. Mathew A, Veliuona MA, DePalma FJ, Cooney RN. Gastrojejunal stricture after gastric bypass and efficacy of endoscopic intervention. Dig Dis Sci 2009;54:1971-8.

15. Ukleja A, Afonso BB, Pimentel R, Szomstein S, Rosenthal R. Outcome of endoscopic balloon dilation of strictures after laparoscopic gastric bypass. Surg Endosc 2008;22:1746-50.

16. Rossi TR, Dynda DI, Estes NC, Marshall JS. Stricture dilation after laparoscopic Roux-en-Y gastric bypass. Am J Surg 2005;189:357-60.

17. Da Costa M, Mata A, Espinós J, Vila V, Roca JM, Turró J, et al. Endoscopic dilation of gastrojejunal anastomotic strictures after laparoscopic gastric bypass. Predictors of Initial Failure. Obes Surg 2010;21:36-41.

18. Alasfar F, Sabnis AA, Liu RC, Chand B. Stricture rate after laparoscopic Roux-en-Y gastric bypass with a 21-mm circular stapler: the Cleveland Clinic experience. Med Princ Pract 2009;18:364-7. 